\title{
Fenologia de Syngonanthus mucugensis Giul. subsp. mucugensis e S. curralensis Moldenke (Eriocaulaceae), nos municípios de Mucugê e Morro do Chapéu, Chapada Diamantina, BA, Brasil
}

\author{
Carlianne Oliveira Cerqueira ${ }^{1,3}$, Ligia Silveira Funch ${ }^{1}$ e Eduardo Leite Borba ${ }^{2}$
}

Recebido em 2/05/2007. Aceito em 31/01/2008

\begin{abstract}
RESUMO - (Fenologia de Syngonanthus mucugensis Giul. subsp. mucugensis e S. curralensis Moldenke (Eriocaulaceae), nos municípios de Mucugê e Morro do Chapéu, Chapada Diamantina, BA, Brasil). Syngonanthus mucugensis subsp. mucugensis e S. curralensis são conhecidas como sempre-vivas por possuírem capítulos que permanecem com a aparência de vivos durante anos. São plantas herbáceas com distribuição agrupada, folhas reunidas em roseta e inflorescências monóicas tipo capítulo, flores alvas e reduzidas. Este estudo apresenta os padrões fenológicos dessas espécies, relacionando-os com fatores abióticos e modo provável de dispersão. As observações foram realizadas mensalmente entre agosto/2002 e setembro/2004, em campo rupestre, nos municípios de Mucugê e Morro do Chapéu, registrando-se presença/ausência das fenofases. A fase vegetativa constitui grande parte do ciclo fenológico, aproximadamente cinco meses, na estação chuvosa (novembro-março). Os escapos levam três a quatro meses do início do desenvolvimento até a presença de capítulos jovens. As flores estaminadas e pistiladas apresentam ântese no início da manhã; as flores estaminadas duram um dia, e o ciclo estaminado cerca de sete dias. As flores pistiladas duram três dias e o ciclo pistilado três a quatro dias. Não ocorre sobreposição temporal das fases estaminada e pistilada em um mesmo capítulo. As duas espécies apresentaram padrão de floração (junho-agosto) e frutificação (julho-novembro) anual, regular, com duração intermediária, na estação seca. A dispersão ocorre ca. 45 dias após a fecundação, estendendose por quatro meses, durante a estação seca e início das chuvas. O tipo de diásporo e a dispersão sazonal sugerem dispersão anemocórica e autocórica.
\end{abstract}

Palavras-chave: Eriocaulaceae, fenologia, sempre-viva, Syngonanthus

\begin{abstract}
The phenology of Syngonanthus mucugensis Giul. subsp. mucugensis and S. curralensis Moldenke (Eriocaulaceae) in Mucugê and Morro do Chapéu municipalities, Chapada Diamantina, Bahia State, Brazil). Syngonanthus mucugensis subsp. mucugensis Giul. and S. curralensis are straw-flowers (sempre-vivas) in which the capitula maintain their appearance and color for many years. These herbaceous plants have a clumped distribution, their leaves form a rosette, and they have small whitish monoecious inflorescences united in a capitulum. The present study examined the phenology of this species, relating it to abiotic environmental factors and probable dispersal modes. The presence/absence of phenophases were observed on a monthly basis from August/2002 to September/2004 in an area of campo rupestre vegetation, in the municipalities of Mucugê and Morro do Chapéu. The vegetative phase is the longest phase of the phenological cycle (approximately five months) and occurs during the rainy season (November through March). Three to four months pass between the emergence of the scapes and the production of the young capitula. The staminate and pistillate flowers undergo anthesis in the early morning; the individual staminate flowers last one day, and the entire staminate cycle lasts seven days. Pistillate flowers last three days, while the pistillate cycle lasts from three to four days. There is no temporal overlap of the staminate and pistillate phases in the same capitulum. The two species demonstrate annual and regular flowering (June-August) and fruiting (JulyNovember), during the dry season. Seed dispersal occurs approximately 45 days after fertilization and extends for four months, during the dry season and the beginning of the rainy season. The type of diaspore produced, the grouped distribution of the species, and the occurrence of seed maturation during the dry season all suggest anemochoric and autochoric dispersal mechanisms.
\end{abstract}

Key words: Eriocaulaceae, phenology, straw-flower, Syngonanthus

\section{Introdução}

A família Eriocaulaceae possui aproximadamente 1.200 espécies, agrupadas em 10 gêneros, com distribuição pantropical, especialmente nas montanhas da Venezuela e em campos rupestres da Cadeia do Espinhaço de Minas Gerais e Bahia, no Brasil (Moldenke 1971; Giulietti \& Pirani 1988; Giulietti \& Hensold 1990). Muitas espécies de Eriocaulaceae são conhecidas popularmente como "sempre-vivas" por seus capítulos

\footnotetext{
1 Universidade Estadual de Feira de Santana, Departamento de Ciências Biológicas, Laboratório de Taxonomia Vegetal, Rodovia BR 116, Km 03, 44031-460 Feira de Santana, BA, Brasil

2 Universidade Federal de Minas Gerais, Instituto de Ciências Biológicas, Departamento de Botânica, Av. Antônio Carlos 6627, $31270-901$ Belo Horizonte, MG, Brasil

3 Autor para correspondência: carliannecerqueira@hotmail.com.
} 
paleáceos, após destacados das plantas, permanecerem com a aparência de vivos durante muitos anos (Giulietti et al. 1996), sendo a maioria destas espécies pertencente ao gênero Syngonanthus, especialmente $S$. sect. Eulepsis. Entre estas estão $S$. mucugensis subsp. mucugensis e S. curralensis, cujos escapos e inflorescências são coletados antes da produção de sementes, secos ao sol e vendidos para a decoração de interiores. Na década de 1980, estas espécies foram amplamente exportadas para os Estados Unidos e Europa (Giulietti et al. 1988). A prática de coleta ainda hoje persiste, o que vem comprometendo a reprodução destas espécies, podendo levá-las à extinção.

Apesar da importância econômica que essas plantas apresentam, pouco se conhece sobre a fenologia dessas espécies, sendo geralmente mencionadas observações de antigos coletores. Em Eriocaulaceae, são conhecidos apenas dois trabalhos que abordam aspectos fenológicos, para as espécies Paepalanthus hilairei, P. polyanthus e P. robustus (Sano 1996) e para Syngonanthus elegans (Scatena et al. 1997). Sendo assim, observa-se a escassez de estudos fenológicos para essa família, o que justifica a realização deste trabalho, onde a fenologia busca analisar a temporalidade dos eventos biológicos cíclicos relacionando-os com forças seletivas bióticas, estacionalidade climática, fatores endógenos e relações filogenéticas (Gentry 1974; Lieth 1974; Bawa 1983; van Schaik et al. 1993; Newstrom et al. 1994; WilliamsLinera \& Meave 2002).

Alguns aspectos têm merecido maior atenção nos estudos da fenologia de floração, como época, freqüência, duração e sincronia, que vêm sendo relacionadas com fatores abióticos, como temperatura, precipitação e umidade (Castellani et al. 1999; Talora \& Morellato 2000; Funch et al. 2002; Williams-Linera \& Meave 2002), com a época de atividade do polinizador (Newstrom et al. 1994) e com o sucesso reprodutivo nas populações (Augspurger 1983; Bawa 1983). Além disso, a partir do estudo de Bullock (1981), vem sendo reconhecida a influência da monoicia no padrão de floração, onde é marcante a diferenciação temporal na expressão de flores unissexuais, determinando padrões reprodutivos dentro e entre populações.

O presente trabalho fez parte de um projeto de conservação e manejo de Eriocaulaceae e outras espécies da Chapada Diamantina ameaçadas de extinção, o qual envolveu estudos de demografia, biologia reprodutiva, variabilidade, propagação e etnobotânica. Sendo assim, este estudo propõe avaliar o comportamento fenológico de S. mucugensis subsp. mucugensis e S. curralensis, discutindo a influência dos fatores abióticos (temperatura, umidade e pluviosidade) e dos modos de polinização e dispersão na determinação dos padrões fenológicos observados em ambas as espécies, contribuindo com informações para o seu manejo e conservação.

\section{Materiais e métodos}

Área de Estudo - O estudo foi realizado nos municípios de Mucugê e Morro do Chapéu, áreas de ocorrência de Syngonanthus mucugensis subsp. mucugensis e S. curralensis, respectivamente, Chapada Diamantina, Bahia, Brasil.

A população de $S$. mucugensis subsp. mucugensis estudada situa-se no Parque Municipal de Mucugê (12 59 '46”S e 41 $20^{\circ}$ "40”W, 959 m de altitude), em vegetação de campo rupestre (Stradmann 1998). A região apresenta solos pouco desenvolvidos, geralmente pobres em nutrientes, rasos, que raramente ultrapassam $50 \mathrm{~cm}$ de profundidade; apresenta clima do tipo Cwb (Köppen 1948), com maior volume de chuvas no final da primavera e no período do verão, com média anual de precipitação em torno de $1.100 \mathrm{~mm}$. A temperatura pode atingir mínimas de $13{ }^{\circ} \mathrm{C}$ no inverno e máximas de $30{ }^{\circ} \mathrm{C}$ nos dias mais quentes do ano, sendo a temperatura média anual de $19,8^{\circ} \mathrm{C}$ (Stradmann 1998).

A população $S$. curralensis estudada encontra-se em áreas adjacentes ao município de Morro do Chapéu, a

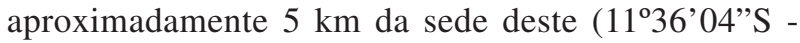
41 $09^{\circ} 47^{\prime \prime} \mathrm{W}, 1.094 \mathrm{~m}$ de altitude), na região conhecida como Tabuleiro dos Tigres. Essa área é classificada como de "tensão ecológica", sendo um contato entre campo rupestre e cerrado (CPRM 1995). Os solos da região apresentam alta acidez e baixa fertilidade. Predomina o tipo climático Cwb (Köppen 1948), com temperatura média do mês mais frio (julho) inferior a $18^{\circ} \mathrm{C}$ e temperatura média do mês mais quente (janeiro) inferior a $22{ }^{\circ} \mathrm{C}$. A temperatura média compensada equivale a $19,7^{\circ} \mathrm{C}$ e a precipitação média anual fica em torno de 800 mm (CPRM 1995).

Espécies estudadas - Syngonanthus mucugensis subsp. mucugensis e $S$. curralensis são espécies herbáceas, com cerca de $40 \mathrm{~cm}$ e $30 \mathrm{~cm}$ de altura, respectivamente, folhas reunidas em roseta e inflorescências monóicas do tipo capítulo, ocorrendo maturação centrípeta das flores e protandria. Os capítulos $S$. mucugensis subsp. mucugensis têm aproximadamente $2,0 \mathrm{~cm}$ de diâmetro e apresentam-se hemisféricos a campanulados, com variação de um a 24 por indivíduo, com nove a 14 séries de brácteas involucrais, que ultrapassam a altura das flores. Nas flores estaminadas, as sépalas são conadas na base e medem 2,1-3,4 mm de comprimento; as pétalas são conadas até a região central e medem 0,8-1,8 mm de comprimento, com pistilódios com aproximadamente $0,8 \mathrm{~mm}$ de comprimento. As flores pistiladas têm sépalas livres e medem 2,5-3,7 mm de comprimento; as pétalas 
são conadas até a região central e medem 1,6-2,3 mm de comprimento; estiletes com 2,1-3,6 mm de comprimento e apêndices com aproximadamente 1,0-1,7 mm de comprimento. O capítulo de $S$. curralensis tem aproximadamente $1,0 \mathrm{~cm}$ de diâmetro e exibe forma hemisférica no início da floração, passando posteriormente a globoso durante o período de dispersão das sementes. Há uma variação de um a 17 capítulos por indivíduo, com oito a 13 séries de brácteas involucrais, não ultrapassando a altura das flores. Nas flores estaminadas, as sépalas são conadas na base e medem 2,1-2,9 mm de comprimento; as pétalas são conadas até região central e medem $0,8-1,7 \mathrm{~mm}$ de comprimento, com pistilódios com aproximadamente $0,5 \mathrm{~mm}$ de comprimento. As flores pistiladas têm sépalas livres e medem 2,3-3,3 mm de comprimento; as pétalas são conadas até região central e medem 1,5-3,2 mm de comprimento; os estiletes têm 1,5-3,2 $\mathrm{mm}$ de comprimento e apêndices com aproximadamente 0,7-1,2 mm de comprimento (Ramos et al. 2005). Em estudo de variabilidade genética e morfológica de Syngonanthus mucugensis, foi constatada a ocorrência de uma subespécie para a região de Rio de Contas e Catolés, descrita como Syngonanthus mucugensis subsp. riocontensis A.C.S. Pereira \& Giul. (Pereira et al. 2007). Sendo assim, o presente trabalho foi realizado com Syngonanthus mucugensis Giul. subsp. mucugensis.

Observações fenológicas - O acompanhamento fenológico de S. mucugensis subsp. mucugensis e $S$. curralensis foi realizado mensalmente, no período de agosto/2002 a setembro/2004, totalizando 26 meses de observações. Foram marcadas aleatoriamente duas transecções de $50 \times 2 \mathrm{~m}$, consistindo em uma amostragem de $100 \mathrm{~m}^{2}$, para cada população estudada, onde foram quantificados, os indivíduos quanto à floração e frutificação. Nas transecções, foram numerados 100 indivíduos adultos, sendo sorteados 20 , os quais foram marcados com placas numeradas, para acompanhamento fenológico, no período de março/2003 a setembro/2004, tendo sido determinadas cinco fenofases, adaptadas de Sano (1996): vegetativa, escapos em desenvolvimento, floração, frutificação, sendo também registrada a fenofase de dispersão. Em seu trabalho, Sano (1996) considerou as fenofases vegetativa, paracládios em desenvolvimento, capítulos fechados, ântese e dispersão.

Foram investigados horário e duração da ântese, sequiência de abertura das flores, duração das fases estaminadas e pistiladas, ocorrência de sobreposição das duas fases e presença de frutos, utilizando-se para esta análise 100 inflorescências de 30 indivíduos de S. mucugensis subsp. mucugensis, durante nove dias consecutivos, no mês de julho/2003, com repetição em agosto/2003. Para S. curralensis, essas análises ocorreram durante dez dias consecutivos, no mês de julho/2003, utilizando-se também 30 indivíduos ao longo da transecção.

As inflorescências foram observadas em campo, com auxílio de uma lupa de mão (20x). O acompanhamento do desenvolvimento das flores até a formação dos frutos foi realizado com base em 20 inflorescências de 10 indivíduos marcados de S. mucugensis subsp. mucugensis e $S$. curralensis.

Durante o período de estudo, outras populações de S. mucugensis subsp. mucugensis situadas no Parque

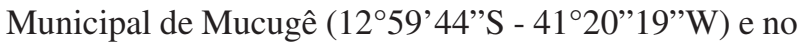
entorno da cidade de Mucugê (Leolbino, $12^{\circ} 58^{\prime} 55^{\prime \prime} \mathrm{S}-41^{\circ} 26^{\prime} 15^{\prime \prime} \mathrm{W}$ e Gobira, $13^{\circ} 05^{\prime} 30^{\prime \prime} \mathrm{S}$ $41^{\circ} 22^{\prime} 09^{\prime}$ 'W) e de $S$. curralensis, situada no entorno da

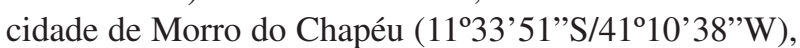
foram visitadas, a fim de se constatar a ocorrência de outras populações próximas às áreas de estudo. Foram observados assistematicamente seus períodos de floração e frutificação.

Dados climatológicos - Para este estudo, os dados climatológicos de precipitação, temperatura e umidade utilizados foram fornecidos pelo Instituto Nacional de Meteorologia (INMET), referentes às estações de Morro do Chapéu e Lençóis, sendo este último município o mais próximo de Mucugê e que possui dados disponíveis. Dados pontuais para Mucugê, coletados pelo "Projeto Sempre Viva", indicam semelhança ao padrão climático de Lençóis.

Análise dos dados - Os padrões de floração e frutificação basearam-se em critérios de frequiência, regularidade e duração do evento (Newstrom et al. 1994).

Utilizando-se o programa Statistica (2000), calculou-se a correlação de Spearman entre o número de indivíduos de Syngonanthus curralensis em floração e frutificação a cada mês e as variáveis climáticas no mesmo período: temperatura média, pluviosidade e umidade.

\section{Resultados e discussão}

Fase vegetativa/Crescimento dos escapos - Em Syngonanthus mucugensis subsp. mucugensis e S. curralensis, os escapos levam em média três meses desde o início do desenvolvimento até a presença de capítulos jovens no ápice, com as brácteas involucrais fechadas. Foi observada uma assincronia na emissão dos escapos em uma mesma população, podendo-se encontrar, indivíduos com crescimento dos escapos e capítulos em desenvolvimento durante cerca de seis meses, em ambas as espécies (Fig. 1A, 2A).

Foi observado que $S$. mucugensis subsp. mucugensis e S. curralensis apresentaram, durante cerca de seis 
meses, apenas estruturas vegetativas (período chuvoso) e nos demais meses do ano, estruturas reprodutivas (período seco), demonstrando uma separação temporal do crescimento vegetativo e da atividade reprodutiva para ambas as espécies que, segundo Bawa et al. (2003), é favorecida pela reprodução na estação seca.

Floração - As flores de S. mucugensis subsp. mucugensis e $S$. curralensis se dispõem no capítulo em três ciclos (séries) de flores estaminadas e três ciclos (séries) de flores pistiladas distribuídos, alternadamente, ao longo do capítulo (Ramos et al. 2005), com as flores mais periféricas amadurecendo primeiro, seguidas das intermediárias e, por fim, das centrais. Em algumas inflorescências de ambas as espécies, foram observados apenas dois ciclos de flores pistiladas. No período estudado, o número de capítulos produzidos por indivíduo variou entre um e 24, em $S$. mucugensis subsp. Mucugensis, e um e 17, em S. curralensis.

A floração de $S$. mucugensis subsp. mucugensis e S. curralensis apresentou-se anual, regular, com duração intermediária, onde a maturação dos ciclos de flores estaminadas e pistiladas ocorreu durante os meses de junho a agosto, com um pico de floração em julho para S. mucugensis subsp. mucugensis e julho-agosto para S. curralensis (Fig. 1B, 2B).

As flores estaminadas de S. mucugensis subsp. mucugensis e $S$. curralensis apresentam ântese entre $8 \mathrm{~h}$ $30 \mathrm{~min}$ e 9 h $30 \mathrm{~min}$. Cada ciclo estaminado dura aproximadamente sete dias, e a cada dia são abertas quatro a 15 flores estaminadas, em $S$. mucugensis subsp. mucugensis, e oito a 19 flores estaminadas, em S. curralensis. As flores pistiladas, em ambas as espécies, apresentam ântese por volta das $8 \mathrm{~h}$. Cada ciclo pistilado tem duração de três a quatro dias, e a cada dia são abertas 15 a 50 flores em $S$. mucugensis subsp. mucugensis e cerca de 30 flores $S$. curralensis, com a maioria abrindo em um mesmo dia. Em ambas as espécies, as flores estaminadas duram um único dia e as flores pistiladas têm duração de três dias, apresentando estigma receptivo apenas no primeiro dia.

Segundo Bawa (1983), em espécies dióicas, as plantas masculinas geralmente apresentam número maior de flores que as femininas e a fase estaminada precede a pistilada. $\mathrm{O}$ autor afirma ainda que a fase pistilada pode ter duração menor que a estaminada. Apesar de monóicas, tais características também foram observadas nas flores das espécies estudadas.

Syngonanthus mucugensis subsp. mucugensis e S. curralensis não apresentaram sobreposição das fenofases no capítulo. Durante o desenvolvimento das flores, foi observado um intervalo na transição da fase pistilada para a estaminada, uma vez que as flores femininas duram três dias, mas se encontraram receptivas apenas no primeiro. Em plantas monóicas com intervalo entre as fases feminina e masculina, o período sem flores elimina a possibilidade de sobreposição entre as fases sexuais, podendo ainda ser fisiologicamente necessário para que ocorra a transformação de uma fase sexual em outra (Bawa 1983).

Ao realizarem estudos reprodutivos experimentais para S. mucugensis subsp. mucugensis, Ramos et al. (2005) observaram que a sobreposição entre as fases pistilada e estaminada é pequena ou inexistente num mesmo indivíduo, apresentando assim comportamento de dioicia temporal, onde a dicogamia marcada desta espécie reduz a autogamia. Esta estratégia também foi observada para as espécies Croton floribundus e C. priscus (L.M. Passos, dados não publicados).

Em S. mucugensis subsp. mucugensis e S. curralensis, os capítulos se comportam como masculinos na maior parte do tempo, uma vez que há maior número de flores estaminadas que pistiladas e a fase estaminada dura mais tempo, não ocorrendo sobreposição temporal das fases estaminadas e pistiladas em um mesmo capítulo (dioicia temporal). As espécies de Syngonanthus estudadas comportam-se temporalmente como plantas dióicas e são polinizadas por pequenos insetos (Ramos et al. 2005) no período mais seco do ano. Tais resultados corroboram os encontrados por Bawa (1980), que observou que a grande maioria das espécies dióicas, em floresta seca na Costa Rica, é polinizada por insetos relativamente pequenos.

O padrão de floração anual e intermediário apresentado por S. mucugensis subsp. mucugensis e S. curralensis foi observado também para S. elegans (Scatena et al. 1997), Paepalanthus hilairei e P. robustus (Sano 1996), o que indica uma tendência a ser investigada na família.

Indivíduos florescendo sincronicamente em uma população atraem maior número de visitantes florais, consequentemente, elevam a frequiência de polinizações (Augspurger 1981). Nas espécies de Eriocaulaceae abordadas neste estudo, foi observado que a abertura sincrônica das flores é um dos fatores que pode aumentar as chances de polinização e, consequentemente, a produção e maturação dos frutos. Lenza \& Oliveira (2005) discutiram o papel desta sincronia de abertura das flores para Tapirira guianensis, aliando a esse fator o display floral maior nas inflorescências masculinas e a maior longevidade das flores femininas como os responsáveis pelo sucesso da polinização nessa espécie.

Neste estudo, foi observada uma alta sincronia quanto ao período de floração dos indivíduos de S. mucugensis subsp. mucugensis e S. curralensis e a época de atividade dos polinizadores relatada por Ramos et al. (2005), o que favoreceu o mecanismo de 
polinização por entomofilia observado para essas espécies, confirmado para $S$. mucugensis subsp. mucugensis através do sucesso reprodutivo de 92,2\% de produção de frutos em polinização aberta (Ramos et al. 2005).

As duas populações de S. mucugensis subsp. mucugensis (Leobino e Gobira) e a população de S. curralensis que foram visitadas durante o período de realização deste estudo, apresentaram comportamento fenológico de floração e frutificação similares aos encontrados para as populações de $S$. mucugensis subsp. mucugensis e $S$. curralensis estudadas.

Em Syngonanthus curralensis, a floração apresentou correlação negativa significativa com a temperatura $\left(r_{S}=-0,795 ; p=0,000001\right)$, não tendo sido encontrada correlação com umidade e pluviosidade (Fig. 2C), indicando que, além dos fatores climáticos, processos endógenos da planta e pressões seletivas bióticas devem influenciar o padrão de floração desta espécie. Richards (1996) afirmou que, em ambientes tropicais sazonais com estação seca bem definida, a maioria das espécies floresce na estação seca. Segundo Janzen (1967), a floração na estação seca seria vantajosa, uma vez que não haveria chuvas pesadas que danificassem as flores. Syngonanthus mucugensis subsp. mucugensis e S. curralensis apresentam flores muito delicadas sendo, conforme Janzen (1967), considerada vantajosa a floração na estação seca.

Frutificação - A frutificação nas duas espécies apresentou-se anual, regular, com duração intermediária, durante os meses de julho a novembro, com picos em agosto a setembro, considerando-se desde indivíduos com frutos imaturos até aqueles que se apresentam dispersando as sementes (Fig. 1B, 2B). O padrão de frutificação anual e intermediário apresentado por S. mucugensis subsp. mucugensis e S. curralensis foi observado também para S. elegans (Scatena et al. 1997), Paepalanthus hilairei e P. robustus (Sano 1996). Entretanto, foi observado que esse padrão apresentou-se mais longo nas espécies de Paepalanthus que nas espécies de Syngonanthus estudadas.

A maturação dos frutos dura cerca de quarenta e cinco dias, quando se inicia a dispersão das sementes, que coincide com períodos de escassez de chuvas nas áreas de estudo (de agosto a dezembro) (Fig. 1A, 2A).

A frutificação de $S$. curralensis apresentou correlação negativa significativa com a pluviosidade $\left(r_{S}=-0,582 ; p=0,0018\right)$ e com a temperatura $\left(r_{S}=-0,546\right.$; $\mathrm{p}=0,0038)$, não tendo apresentado correlação com a umidade (Fig. 2C), demonstrando a influência dos fatores ambientais na área de estudo sobre a espécie estudada. Koptur et al. (1988) e Morellato \& Leitão Filho (1990) sugerem que, em ambientes sazonais, os fatores climáticos têm maior influência sobre as fenofases do que em ambientes pouco sazonais. A umidade, por sua vez, provavelmente não apresentou correlação com a frutificação, por esta se manter com uma curta variação durante o período de estudo.

Syngonathus mucugensis subsp. mucugensis e S. curralensis apresentaram tanto o padrão de floração como o de frutificação sazonal, reproduzindo-se apenas na estação seca. Miranda (1995) observou que espécies anemocóricas que apresentaram frutos maduros na estação seca (setembro a dezembro), também apresentam um comportamento sazonal. A floração e frutificação de Paepalanthus hilairei, P. polyanthus e P. robustus ocorrem no período chuvoso (Sano 1996), ao contrário de Syngonanthus elegans, que apresenta sua fase reprodutiva no período mais seco (Scatena et al. 1997).

A escassez hídrica observada parece favorecer o desenvolvimento reprodutivo de $S$. mucugensis subsp. mucugensis e $S$. curralensis, uma vez que ocorreram floração e frutificação da maioria dos indivíduos no período mais seco dos anos observados, corroborando a idéia de que o estresse hídrico é o maior estímulo para a floração durante a estação seca (Borchert 1980; 1983).

Dispersão - Os indivíduos de S. curralensis e S. mucugensis subsp. mucugensis apresentaram distribuição agrupada nas populações estudadas, com grande número de plântulas próximas aos indivíduos adultos, o que reflete uma dispersão de sementes a curtas distâncias. As duas espécies apresentaram fruto do tipo cápsula, loculicida, de coloração castanho-escura, com cerca de 1,5 mm de diâmetro. São produzidas três sementes por fruto, muito leves, elípticas, com testa de coloração castanho avermelhada e cerca de $0,5 \mathrm{~mm}$ de diâmetro.

O tipo de diásporo de ambas as espécies apresenta características relacionadas com anemocoria, como sementes leves e diminutas, dispersas na estação seca e em áreas abertas, podendo ser classificado, segundo van der Pijl (1982), como pulverulento. Segundo Augspurger (1983), esse diásporo seria do tipo "não-classificado", por não apresentar desenho morfológico diretamente relacionado com a dispersão pelo vento, embora seu peso e tamanho indiquem dispersão anemocórica.

Os diásporos de ambas as espécies não apresentam estruturas secundárias, como papus e alas, que favorecem a dispersão a longa distância, entretanto, as características morfológicas, como peso e tamanho, poderiam favorecer este tipo de dispersão. Sendo assim, sugerimos que a dispersão nestas espécies pode ocorrer por meio de anemocoria, onde as cápsulas se abrem, deixando as sementes expostas entre as brácteas do capítulo. As sementes são dispersas quando o vento 
atua, fazendo movimentar os escapos - que são bastante delicados e suscetíveis à ação dos ventos - entretanto, a distância alcançada por esses diásporos não parece ser muito grande, sendo necessários maiores estudos.

Por outro lado, a distribuição agrupada dos indivíduos nas populações e o grande número de plântulas próximas a indivíduos adultos sugerem também a possibilidade de dispersão autocórica para S. curralensis e S. mucugensis subsp. mucugensis. Após a liberação das sementes, os capítulos tendem a inclinar-se em direção ao solo, sendo encontrados caídos a partir do mês de novembro, quando ocorre a formação de novas folhas na roseta.

A maturação dos frutos de $S$. mucugensis subsp. mucugensis e $S$. curralensis no final da estação seca e início da chuvosa pode estar relacionada com as condições favoráveis para a germinação, devido à maior disponibilidade hídrica, favorecendo o desenvolvimento do sistema radicular durante a estação úmida. Segundo Morellato \& Leitão Filho (1992), a maturação dos frutos na estação seca, pouco antes das chuvas, aumenta a probabilidade de germinação e crescimento das plântulas. Scatena et al. (1997) observaram dispersão das sementes de Syngonanthus elegans no início do período chuvoso, sugerindo que essa época é favorável à germinação das sementes e estabelecimento das plântulas desta espécie.

As sementes das espécies de Paepalanthus estudadas por Sano (1996) caem muito próximas à planta-mãe, promovendo um grande agrupamento de indivíduos jovens, o que reforça a idéia de autocoria
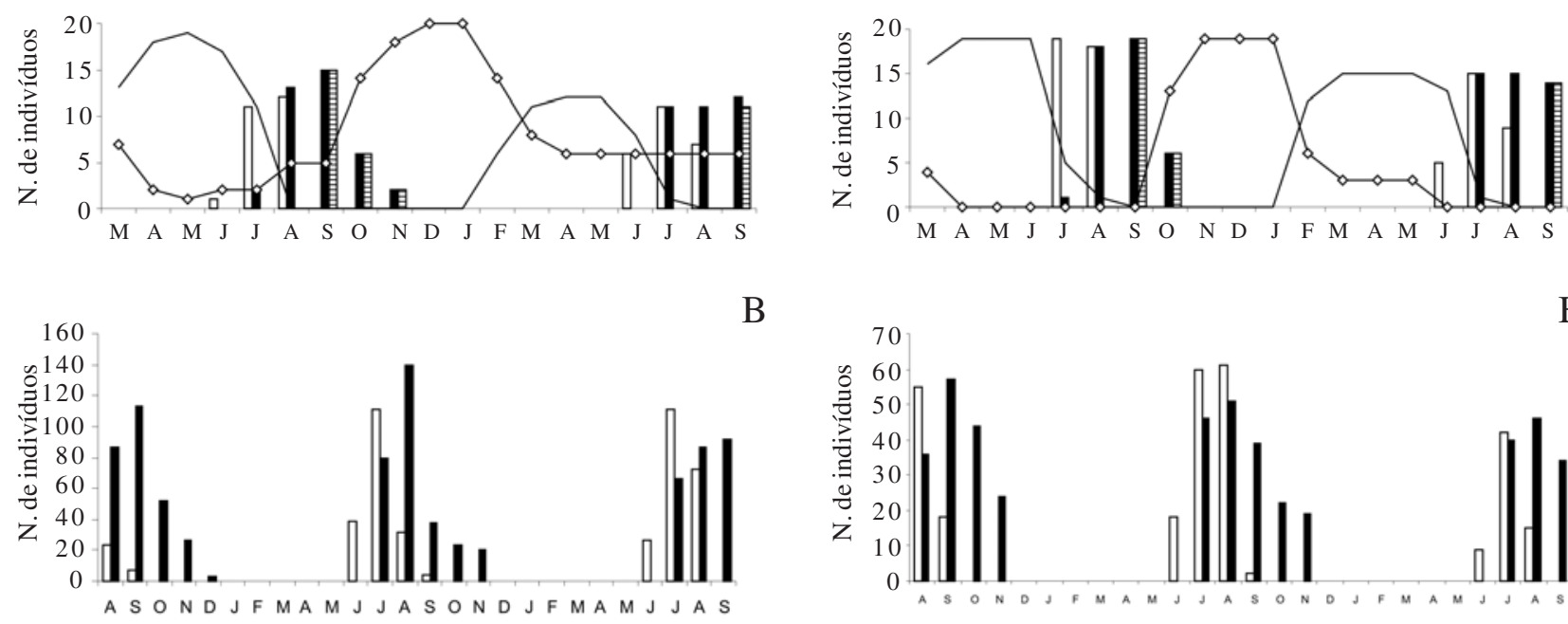

B

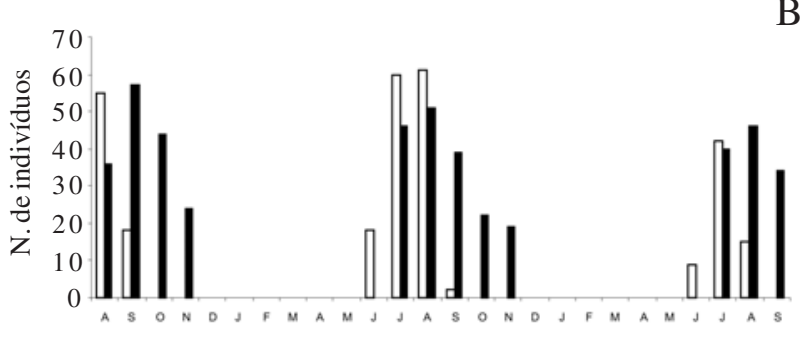

$\mathrm{C}$

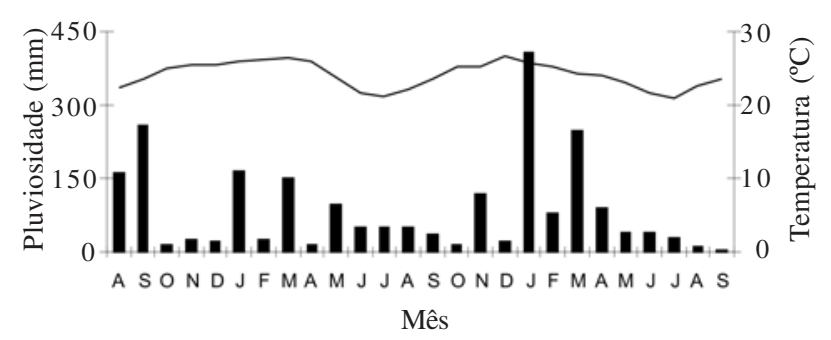

Figura 1. Fenofases observadas para os indivíduos marcados $(\mathrm{n}=20)$ de Syngonanthus mucugensis subsp. mucugensis (A) no período de março/2003 a setembro/2004; número total de indivíduos de S. mucugensis subsp. mucugensis, na transecção de $50 \times 2 \mathrm{~m}$, em floração e frutificação no período de agosto/2002 a setembro/2004, no município de Mucugê, BA, Brasil (B) e dados climáticos fornecidos pelo Instituto Nacional de Meteorologia (INMET) para o município de Lençóis, BA, Brasil (C). (A. $\square$ = Floração; $\square=$ Frutificação; $\boxminus=$ Dispersão; $-\diamond-=$ vegetativa; $-=$ escapos. B. $\square=$ Floração;

$\mathbf{\square}=$ Frutificação. C. $\mathbf{\square}=$ Pluviosidade; - = Temperatura).

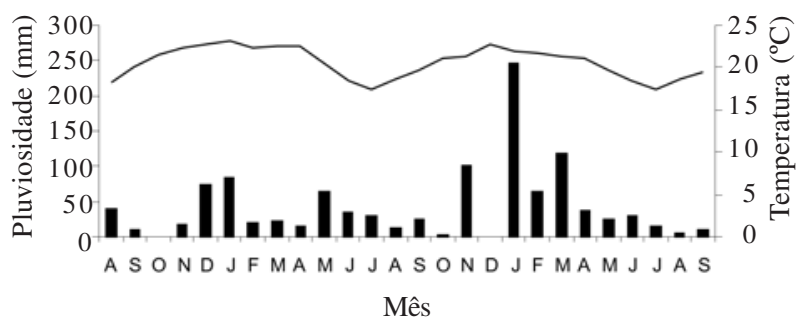

Figura 2. Fenofases observadas para os indivíduos marcados $(\mathrm{n}=20)$ de Syngonanthus curralensis (A) no período de março/2003 a setembro/2004; número total de indivíduos de $S$. curralensis, na transecção de $50 \times 2 \mathrm{~m}$, em floração e frutificação no período de agosto/2002 a setembro /2004, no município de Morro do Chapéu, BA, Brasil (B) e dados climáticos fornecidos pelo Instituto Nacional de Meteorologia (INMET) para o município de Morro do Chapéu, BA, Brasil (C). (A. $\square=$ Floração; $\mathbf{\square}=$ Frutificação; $\boxminus=$ Dispersão; $-\diamond-=$ vegetativa; $-=$ escapos. B. $\square=$ Floração; $\mathbf{\square}=$ Frutificação. C. $\mathbf{\square}=$ Pluviosidade; $-=$ Temperatura). 
nessas espécies. Scatena et al. (1997) observaram que os capítulos de Syngonanthus elegans, ao inclinarem-se em direção ao solo, com auxílio dos ventos fortes, também apresentam dispersão autocórica.

A relação entre baixa umidade e abertura dos frutos, observada nas espécies de Eriocaulaceae estudadas, é resultado da dessecação de suas cápsulas loculicidas, que consequentemente dispersam as sementes diminutas durante a estação seca. Essa relação vem sendo relatada como importante fator no processo de dessecação que acompanha a maturação dos diásporos de espécies de Leguminosae, Bombacaceae, Asclepiadaceae e Bignoniaceae (Janzen 1967).

Fenologia para indivíduos marcados - Em relação aos indivíduos marcados $(\mathrm{n}=20)$, foi observado que, no período de março/2003 a fevereiro/2004, ocorreu floração e frutificação em 15 indivíduos de $S$. mucugensis subsp. mucugensis não tendo sido registrada ocorrência de morte; em S. curralensis, houve desenvolvimento reprodutivo de 19 indivíduos durante o período de estudo (Fig. 1A, 2A). Ao final das observações (setembro/2004), foram registradas, entre os indivíduos marcados, três mortes para $S$. mucugensis subsp. mucugensis e seis para S. curralensis. Foi observado que, em ambas as espécies, a floração ocorreu nos meses de junho a agosto e a frutificação ocorreu entre julho e outubro para S. curralensis, estendendo-se um pouco mais para S. mucugensis subsp. mucugensis, julho a novembro.

Em S. mucugensis subsp. mucugensis foi observado que em dois dos indivíduos marcados que morreram ocorreu desenvolvimento de novas rosetas em agosto/2004. Acredita-se que as sementes dispersas tenham encontrado ambiente propício para germinação entre as folhas velhas dos indivíduos mortos, ou tenha ocorrido brotamento. A produção de novas rosetas não foi observada para os indivíduos mortos de $S$. curralensis.

O microhabitat particular em que vivem as espécies pode ser um fator importante na variabilidade fenológica entre indivíduos (Williams-Linera \& Meave 2002), fato este que pode justificar a não floração de alguns indivíduos de S. mucugensis subsp. mucugensis e S. curralensis marcados. Nas áreas estudadas, foram observados indivíduos com vegetação herbácea associada (área sombreada) e indivíduos isolados (maior exposição à luz solar). Embora não tenham sido registradas diferenças no período reprodutivo desses indivíduos e na época de desenvolvimento dos escapos, maiores investigações podem auxiliar no entendimento sobre a ausência de floração de alguns deles, durante o período do estudo.

Os resultados do presente estudo indicam que as espécies S. mucugensis subsp. mucugensis e S. curralensis apresentam floração, frutificação e dispersão sazonal, havendo um ajuste destas fenofases com a estação seca. A dispersão no período de transição entre a estação seca e início da chuvosa pode ser uma estratégia fenológica, favorecendo a germinação e estabelecimento das plântulas no período de maior disponibilidade hídrica.

O número de inflorescências produzidas em S. mucugensis subsp. mucugensis e $S$. curralensis sugere capacidade de manutenção das populações estudadas e sucesso na polinização, diminuindo o risco de extinção sofrido por ambas as espécies.

Estudos fenológicos sobre Syngonanthus mucugensis ssp. mucugensis e S. curralensis são importantes no sentido de poderem contribuir para futuros trabalhos que visem seu manejo e cultivo, e, consequentemente, auxiliarão na sua preservação, uma vez que essas espécies são endêmicas da região de Mucugê e Morro do Chapéu, respectivamente, e exploradas comercialmente.

\section{Agradecimentos}

Os autores agradecem a Delmar L. Alvim e Oremildes A. Oliveira, pela ajuda nos trabalhos de campo; a Roy Funch, pela ajuda com o abstract; e aos revisores anônimos, pelas valiosas críticas e sugestões. Este trabalho foi financiado pelo Fundo Nacional de Meio Ambiente (FNMA \#75/2001). Caroline O. Cerqueira recebeu bolsa de estudos da Fundação de Amparo à Pesquisa do Estado da Bahia (FAPESB). E.L. Borba tem apoio do $\mathrm{CNPq}(\mathrm{PQ} 2)$.

\section{Referências bibliográficas}

Augspurger, C.K. 1981. Reproductive synchrony of tropical plant: experimental effects of pollinators and seed predators on Hybanthus prunifolius (Violaceae). Ecology 62: 775-788.

Augspurger, C.K. 1983. Phenology, flowering synchrony, and fruit set of six neotropical shrubs. Biotropica 15: 257-267.

Bullock, S. 1981. Notes on the phenology in inflorescences and pollination of some rain forest palms in Costa Rica. Principes 25: 101-105.

Bawa, K.S. 1980. Evolution of dioecy in flowering plants. Annual Review of Ecology and Systematics 11: 15-39.

Bawa, K.S. 1983. Patterns of flowering in tropical plants. Pp. 394-410. In: C.E. Jones \& R.J. Little (eds.). Handbook of experimental pollination biology. Van Nostrand Reinhold, New York.

Bawa, K.S.; Kang, H. \& Grayum, M.H. 2003. Relationships among time, frequency, and duration of flowering in tropical rain forest trees. American Journal of Botany 90: 877-887.

Borchert, R. 1980. Phenology and ecophysiology of tropical trees: Erytrina poeppigiana O.F. Cook. Ecology 61: 1065-1074.

Borchert, R. 1983. Phenology and control of flowering in tropical trees. Biotropica 15: 81-89.

Castellani, T.T.; Caus, C.A. \& Vieira, S. 1999. Fenologia de uma comunidade de duna frontal no sul do Brasil. Acta Botanica Brasilica 13: 99-144. 
CPRM - Companhia de Pesquisa de Recursos Minerais. 1995. Projeto mapas municipais do município de Morro do Chapéu, Bahia. Salvador.

d'Eça-Neves, F.F. \& Morellato, L.P.C. 2004. Métodos de amostragem e avaliação utilizados em estudos fenológicos de florestas tropicais. Acta Botanica Brasilica 18: 99-108.

Funch, L.S.; Funch, R. \& Barroso, G.M. 2002. Phenology of gallery and montane forest in the Chapada Diamantina, Bahia, Brazil Biotropica 34: 40-50.

Gentry, A.H. 1974. Flowering phenology and diversity in tropical Bignoniaceae. Biotropica 6: 48-64.

Giulietti, A.M. \& Pirani, J.R. 1988. Patterns of geographic distribution of some plant species from the Espinhaço Range, Minas Gerais and Bahia, Brazil. Pp. 39-69. In: W.R. Heyer \& P. E. Vanzolini (eds.). Proceedings of a Workshop on neotropical distribution patterns. Rio de Janeiro.

Giulietti, N.; Giulietti, A.M.; Pirani, J.R. \& Menezes, N.L. 1988. Estudos em sempre-vivas: importância econômica do extrativismo em Minas Gerais, Brasil. Acta Botanica Brasilica 1: 179-193 supl.

Giulietti, A.M. \& Hensold, N. 1990. Padrões de distribuição geográfica dos gêneros de Eriocaulaceae. Acta Botanica Brasilica 4: $133-158$.

Giulietti, A.M.; Wanderley, M.G.L.; Longhi-Wagner, H.M.; Pirani, J.R. \& Parra, L.R. 1996. Estudos em "sempre-vivas": taxonomia com ênfase nas espécies de Minas Gerais, Brasil. Acta Botanica Brasilica 10: 329-377

Köppen, W. 1948. Climatologia com un estudio de los climas de la Tierra (traduzido por P.R.H. Peres). Mexico, Fondo de Cultura Economica.

Janzen, D.H. 1967. Synchronization of sexual reproduction of trees within the dry season in central América. Evolution 21: 620-637.

Koptur, S.; Haber, W.A.; Frankie, G.W. \& Baker, H.G. 1988. Phenological studies of shrub and treelet species in tropical cloud forests of Costa Rica. Journal of Tropical Ecology 4: 323-346.

Lenza, E. \& Oliveira, P.E. 2005. Biologia reprodutiva de Tapirira guiananesis Aubl. (Anacardiaceae), uma espécie dióica em mata de galeria do Triângulo Mineiro, Brasil. Revista Brasileira de Botânica 28: 179-190.

Lieth, H. 1974. Purpose of a phenology book. Pp. 2-19. In: H. Lieth (ed.). Phenology and seasonality modeling. Berlin. Springer.

Miranda, I.S. 1995. Fenologia do estrato arbóreo de uma comunidade de cerrado em Alter-do-chão, PA. Revista Brasileira de Botânica 18: 235-240.

Moldenke, H.N. 1971. A fifth sumary of the Verbenaceae, Avicenniaceae, Stilbaceae, Dicrastylidaceae, Symphoremaceae, Nyctanthaceae and Eriocaulaceae of the world as to valid taxa, geographic distribution and synonymy. $2 \mathrm{v}$. Ann Arbor. Braun-Brumfield.
Morellato, L.P.C. \& Leitão Filho, H.F. 1990. Estratégias fenológicas de espécies arbóreas em floresta mesófila na Serra do Japi, Jundiaí, SP. Revista Brasileira de Biologia 50: 163-173.

Morellato, L.P.C. \& Leitão Filho, H.F. 1992. Padrões de frutificação e dispersão na Serra do Japi. Pp. 112-140. In: L.P.C. Morellato (ed.). História natural da Serra do Japi: ecologia e preservação de uma área florestal no sudeste do Brasil. Campinas, Editora da Unicamp/Fapesp.

Newstrom, L.E.; Frankie, G.W. \& Baker, H.G. 1994. A new classification for plant phenology basead on flowering patterns in lowland tropical rain forest trees at La Selva, Costa Rica. Biotropica 26: 141-159.

Ramos, C.O.C.; Borba, E.L. \& Funch, L.S. 2005. Pollination in Brazilian Syngonanthus (Eriocaulaceae) Species: Evidence for Entomophily Instead of Anemophily. Annals of Botany 96: 387-397.

Richards, P.W. 1996. The Tropical Rain Forest an ecological study. $2^{\text {nd }}$ ed. United Kingdom, Cambridge University Press.

Sano, P.T. 1996. Fenologia de Paepalanthus hilairei Koern., P. polyanthus (Bong.) Kunth e P. robustus Silveira: Paepalanthus sect. Actinocephalus Koern. - Eriocaulaceae. Acta Botanica Brasilica 10: $317-328$.

Sarmiento, G. \& Monasterio, M. 1983. Life forms and phenology. Pp. 79-108. In: F. Bourliere (ed.). Ecosystems of the word: tropical savannas. Elsevier Science, Amsterdam.

Scatena, V.L.; Lima, A.A.A. \& Lemos Filho, J.P. 1997. Aspectos fenológicos de Syngonanthus elegans (Bong.) Ruhl. (Eriocaulaceae) da Serra do Cipó, MG, Brasil. Arquivos Biologicos Tecnologicos 40: 153-167.

Statsoft Inc. 2000. Statistica For Windows [Computer program manual]. Tulsa, OK: StatSoft, Inc., 2300 East 14th Street, Tulsa, OK 74104, phone: (918) 749-1119, fax: (918) 749-2217, email: info@statsoft.com,WEB: http://www.statsoft.com

Stradmann, M.T.S. 1998. Plano de Manejo do Parque Municipal de Mucugê. Mucugê, Prefeitura Municipal de Mucugê.

Talora, D.C. \& Morellato, L.P.C. 2000. Fenologia de espécies arbóreas em floresta de planície litorânea do sudeste do Brasil. Revista Brasileira de Botânica 23: 13-26.

Van Der Pijl, L. 1982. Principles of dispersal in higher plants. $3^{\text {rd }}$ ed. Berlin, Springer-Verlag.

Van Shaick, C.P.; Terborgh, J.W. \& Wright, S.J. 1993. The phenology of tropical forests: adaptative significance and consequences for primary consumers. Annual Rewiew of Ecology and Systematics 24: 353-377.

Williams-Linera, G. \& Meave, J. 2002. Patrones fenológicos. Pp. 407-431. In: M.R. Guariguata \& G.H. Kattan (eds.). Ecología y conservación de bosques neotropicales. Costa Rica, Libro Universitario regional. 